\title{
Spectral Structure of the Pygmy Dipole Resonance
}

\author{
A. P. Tonchev, ${ }^{1,2}$ S. L. Hammond, ${ }^{3,2}$ J. H. Kelley, ${ }^{4,2}$ E. Kwan, ${ }^{1,2}$ H. Lenske, ${ }^{5}$ G. Rusev, ${ }^{1,2}$ W. Tornow, ${ }^{1,2}$ and N. Tsoneva ${ }^{5,6}$ \\ ${ }^{1}$ Duke University, Durham, North Carolina 27708-0308, USA \\ ${ }^{2}$ Triangle Universities Nuclear Laboratory, Durham, North Carolina 27708-0308, USA \\ ${ }^{3}$ University of North Carolina at Chapel Hill, Chapel Hill, North Carolina 27599-3255, USA \\ ${ }^{4}$ North Carolina State University, Raleigh, North Carolina 27695-8202, USA \\ ${ }^{5}$ Institut für Theoretische Physik, Universität Gießen, Gießen, D-35392, Germany \\ ${ }^{6}$ Institute for Nuclear Research and Nuclear Energy, 1784 Sofia, Bulgaria
}

(Received 23 July 2009; published 18 February 2010)

\begin{abstract}
High-sensitivity studies of $E 1$ and $M 1$ transitions observed in the reaction ${ }^{138} \mathrm{Ba}\left(\vec{\gamma}, \gamma^{\prime}\right)$ at energies below the one-neutron separation energy have been performed using the nearly monoenergetic and $100 \%$ linearly polarized photon beams of the $\mathrm{HI} \vec{\gamma} \mathrm{S}$ facility. The electric dipole character of the so-called "pygmy" dipole resonance was experimentally verified for excitations from 4.0 to 8.6 MeV. The fine structure of the $M 1$ "spin-flip" mode was observed for the first time in $N=82$ nuclei.
\end{abstract}

DOI: 10.1103/PhysRevLett.104.072501

In stable and unstable neutron-rich nuclei a resonancelike concentration of dipole strength is observed at excitation energies around the neutron-separation energy [1-10]. This clustering of strong dipole transitions has been named the pygmy dipole resonance (PDR). In hydrodynamic and collective approaches, it was suggested that an oscillation of a small portion of neutron-rich nuclear matter relative to the rest of the nucleus is responsible for the generation of pygmy resonances [11,12]. Further, in microscopic models based on the quasiparticle-random-phase approximation, relativistic (RQRPA) and nonrelativistic (QRPA), the position and the distribution of the PDR have been investigated [13-16]. From the analysis of transition densities, the unique behavior of the PDR mode is revealed, making it distinct from the well-known giant dipole resonance (GDR) [17]. The systematic studies of the PDR over isotonic and isotopic chains of nuclei indicate a correlation between the observed total $B(E 1)$ strength of the PDR and the neutron-to-proton ratio $N / Z[5,8,14,15]$. In addition, it has been suggested that the PDR is independent of the type of nucleon excess (neutron or proton) $[13,15]$.

The existence of the PDR mode near the neutron threshold has also important astrophysical implications. For example, the reaction rate of the $(\gamma, n)$ and $(n, \gamma)$ reactions in explosive nucleosynthesis of certain neutron deficient heavy nuclei may be significantly enhanced by the PDR [18]. Furthermore, for very neutron-rich exotic nuclei, the PDR is an important topic of study at the new generation of radioactive ion beam facilities [19].

In many cases the interpretation of the PDR excitation is based on the assumption of negative parity for the majority of the $J=1$ states. However, there has not been a systematic experimental verification that all the dipole states observed in the entire PDR region are indeed $1^{-}$states. The parity was measured directly in certain energy intervals, e.g., off-axis bremsstrahlung or Compton polarimetry [20]. The advantage of using $100 \%$ linearly polarized
PACS numbers: 21.10.Hw, 21.60.-n, 23.20.En, 24.70.+s

photon beams for parity identification has been recently demonstrated [21-24], which opens new opportunities for unveiling the character of the nuclear dipole response.

On the other hand, in heavy mass nuclei there should be $M 1$ strength located in the same excitation energy region as the PDR, i.e., in the low-energy tail of the GDR [17]. A major experimental problem is to distinguish $M 1$ and $E 1$ strengths, since the former is highly fragmented at these energies [17]. In order to unambiguously discriminate between these different dipole excitation modes, the spin and parity of the involved states must be known.

In the present Letter, we report first on the experimental determination of the character of the PDR as a predominantly $E 1$ mode of excitation. Furthermore, the fine structure of the dominant $E 1$ and dispersed $M 1$ strength distributions below the particle separation energy is determined. Finally, the dynamics of the PDR as an interplay between isoscalar and isovector excitation modes is revealed. Our experimental study focuses on the ${ }^{138} \mathrm{Ba}\left(\vec{\gamma}, \gamma^{\prime}\right)$ reaction below the neutron-separation energy of about 8.6 MeV using the nearly monoenergetic and $100 \%$ linearly polarized photon beams of the HighIntensity $\gamma$-Ray Source $(\mathrm{HI} \vec{\gamma} \mathrm{S})$ facility [25].

The monoenergetic photon beams were produced by intracavity Compton backscattering of free-electron-laser light from electron beam bunches and collimated by a lead collimator of length $30.5 \mathrm{~cm}$ with a cylindrical hole of $1.9 \mathrm{~cm}$ diameter. The photon flux on target exceeds $1000 \gamma \mathrm{eV}^{-1} \mathrm{~s}^{-1}$ within an energy spread of about $3 \%$. After proper attenuation, the energy distribution of the photon beam was measured with a $123 \%$ high-purity germanium (HPGe) detector placed in the beam. The photon flux was measured by Compton scattering of the beam from a $1.0 \mathrm{~mm}$ thick copper plate, positioned $3 \mathrm{~m}$ behind the barium target.

The target consisted of $4.2905 \mathrm{~g}$ of $\mathrm{BaCO}_{3}$ powder enriched to $99.68 \%$ in ${ }^{138} \mathrm{Ba}$. It was placed into an evacu- 
ated plastic tube at the center of an array of five largevolume HPGe detectors. These detectors were arranged around the target at three positions: $(\theta, \phi)=$ $\left(90^{\circ}, 90^{\circ}\right.$, and $\left.270^{\circ}\right),\left(90^{\circ}, 0^{\circ}\right.$, and $\left.180^{\circ}\right)$, and $\left(135^{\circ}\right.$, $0^{\circ}$ ), where $\theta$ is the polar angle of the outgoing radiation with respect to the horizontally polarized incoming photon beam, and $\phi$ is the azimuthal angle measured from the polarization plane. This detector configuration allows for unambiguous determination of $E 1, M 1$, and $E 2$ transitions regardless of the ground-state spin.

The photon scattering spectra at three different angles relative to the linearly polarized $\mathrm{HI} \vec{\gamma} \mathrm{S}$ beam is shown in Fig. 1. All peaks seen in these spectra represent groundstate transitions of excited states in ${ }^{138} \mathrm{Ba}$. The dotted lines between the top and the center spectrum connect the transitions present in the vertical and backward-angle inplane detectors, but not seen in the horizontal detectors at $90^{\circ}$. Hence, from the azimuthal intensity patterns for elastic scattering of linearly polarized photons, one can unambiguously assign pure $E 1$ excitations to the observed transitions [26,27]. On the other hand, the bottom spectrum shows transitions present in the horizontal and backwardangle detectors, but not in the vertical detectors. These transitions correspond to pure $M 1$ excitations.

In this way, a total of 18 measurements were carried out, covering the entire energy region from 4.0 to $8.6 \mathrm{MeV}$. Only transitions with phonon width greater than $1 \mathrm{meV}$ were resolved. Our measurements confirm the previous parity assignments in one of the pioneering experiments at $\mathrm{HI} \vec{\gamma} \mathrm{S}$ between 5.5-6.5 MeV [21] and the dipole character of the states measured in Refs. [1,5]. However, higher experimental sensitivity allowed us to observe 87 new dipole states in ${ }^{138} \mathrm{Ba}$. Most of these states are at energies above $6.5 \mathrm{MeV}$ and have relatively small strength. The

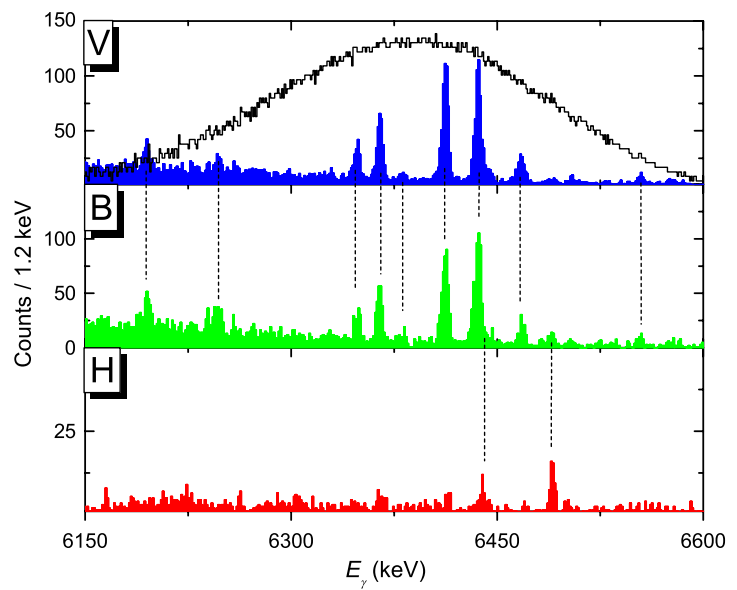

FIG. 1 (color online). Gamma-ray spectra of the ${ }^{138} \mathrm{Ba}\left(\vec{\gamma}, \gamma^{\prime}\right)$ reaction observed in two vertical detectors $(V)$ positioned at $(\theta$, $\phi)$ of $\left(90^{\circ}, 90^{\circ}\right)$ and $\left(90^{\circ}, 270^{\circ}\right)$, one in-plane backward-angle detector $(B)$ positioned at $(\theta, \phi)$ of $\left(135^{\circ}, 0^{\circ}\right)$, and two horizontal detectors $(H)$ positioned at $(\theta, \phi)$ of $\left(90^{\circ}, 0^{\circ}\right)$ and $\left(90^{\circ}\right.$, $\left.180^{\circ}\right)$. The energy distribution of the photon beam with a centroid at $6400 \mathrm{keV}$ is shown in the top panel. summed $E 1$ transition strength from 4.0 to $8.6 \mathrm{MeV}$ is $\sum B(E 1) \uparrow=0.96(18) e^{2} \mathrm{fm}^{2}$, corresponding to $1.3 \%$ of the energy-weighted sum rule (EWSR).

Equally important are the concentrations of $M 1$ dipole states which have been observed for the first time in ${ }^{138} \mathrm{Ba}$. They are clustered around $6.5 \mathrm{MeV}$ and close to the neutron-separation energy. The summed $M 1$ transition strength from 4.0 to $8.6 \mathrm{MeV}$ is $\Sigma B(M 1) \uparrow=2.5(6) \mu_{N}^{2}$, contained in 19 newly observed resonances. About $1.0 \mu_{N}^{2}$ of this strength is localized within a doorway state at $6.5 \mathrm{MeV}$. The centroid energy of these states corresponds to $E \approx 35 A^{-1 / 3} \mathrm{MeV}$.

The $100 \%$ polarized and nearly monoenergetic photon beam allows us not only to determine unambiguously the electromagnetic character of the transitions, but it also enables a closer examination of the dynamics of the decay pattern in the PDR region. First, it should be noted that no sizable branching transitions to low-lying excited states (greater than 2\%) have been observed. This fact is confirmed by the microscopic calculations presented below, suggesting that the elastic dipole transitions are due to almost pure neutron particle-hole states decoupled from the GDR. According to the calculated structure of the wave functions of QRPA $1^{-}$states and corresponding proton and neutron transition densities, they have been associated with the PDR $[13,15]$.

Second, at excitation energies close to the neutronseparation energy, the density of complex configurations like $2 p-2 h, 3 p-3 h$, etc., becomes higher. Accordingly, the inelastic part of the absorption cross section increases with the energy and smoothly merges with the low-energy tail of the GDR. The inelastic cross section was also measured directly by the decay of the low-lying $2^{+}$states to the ground state, which are known to collect most of the inelastic transitions cascading from higher excitation energies. The elastic $\left(\sigma_{\gamma \gamma}\right)$, inelastic $\left(\sigma_{\gamma \gamma^{\prime}}\right)$, and photoabsorption $\left(\sigma_{\gamma}=\sigma_{\gamma \gamma}+\sigma_{\gamma \gamma^{\prime}}\right)$ cross sections for the ${ }^{138} \mathrm{Ba}\left(\vec{\gamma}, \gamma^{\prime}\right)$ reaction below the neutron-separation energy are shown in the upper panel of Fig. 2. These cross sections include transitions from both $1^{-}$and $1^{+}$states. The elastic cross section, which is due to ground-state transitions, shows resonancelike structures and dominates the nuclear dipole response up to the neutron-separation energy. In contrast, the inelastic cross section which is due to statistical decays, shows an exponential increase with excitation energy. The deduced photoabsorption cross section $\left(\sigma_{\gamma}\right)$ is shown in the lower panel of Fig. 2 in comparison to experimental data for the ${ }^{138} \mathrm{Ba}(\gamma, x n)$ reaction in the GDR region [28]. As can be seen, for certain energies below the neutron-separation energy $\left(S_{n}\right)$ the photoabsorption cross section has values larger than the extrapolation of the tail of the GDR implies.

We have performed microscopic calculations of lowenergy electric $1^{-}$and magnetic $1^{+}$dipole states in ${ }^{138} \mathrm{Ba}$, respectively, within the quasiparticle-phonon model (QPM) approach [29] based on density functional theory, 


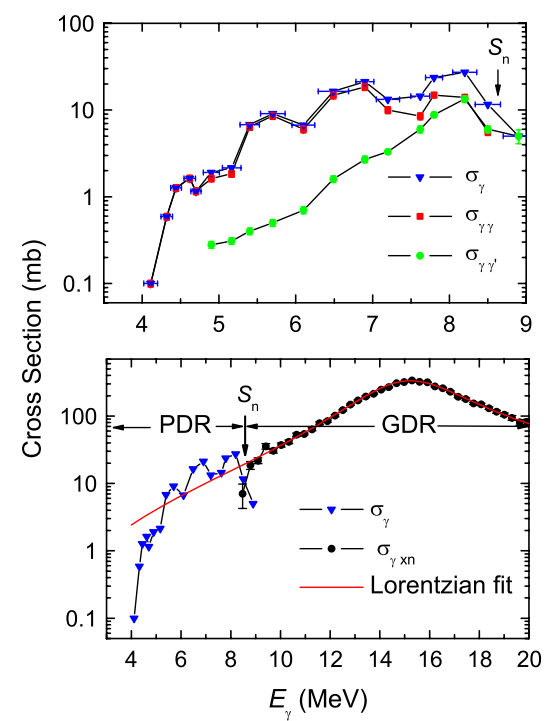

FIG. 2 (color online). Upper panel: Elastic $\left(\sigma_{\gamma \gamma}\right)$, inelastic $\left(\sigma_{\gamma \gamma^{\prime}}\right)$, and total absorption cross sections $\left(\sigma_{\gamma}\right)$ in ${ }^{38} \mathrm{Ba}$ below the one-neutron-separation energy $\left(S_{n}=8.6 \mathrm{MeV}\right)$ averaged over the beam energy spread of about $3 \%$. The actual beam energy spread is shown as horizontal bars. Lower panel: the present total absorption cross section $\left(\sigma_{\gamma}\right)$ is combined with the $(\gamma, x n)$ data from the GDR [28].

as discussed in detail in Refs. [14,15]. In the calculations of the $M 1$ strength, an effective spin giromagnetic factor $g_{s}^{\text {eff }}=0.6 g_{s}$ is implemented. The two-quasiparticle (2QP) QRPA calculations lead to a sequence of $1^{-}$states in the energy range $E^{*}=6.0-8.2 \mathrm{MeV}$. The structure of these states is strongly dominated by the $2 \mathrm{QP}$ neutron configurations formed by quasiparticle states from the $3 s$, $2 d, 1 g$ shells and the $3 p, 2 f$ shells, respectively. The transition densities reveal an oscillation pattern typical for PDR modes [15]. The wave function of the magnetic $1^{+}$states is dominated by $2 \mathrm{QP}$ spin-flip states. The lowest QRPA $1_{1}^{+}$state is found at $E^{*}=4.28 \mathrm{MeV}$ with a transition strength $B\left(M 1\right.$; g.s. $\left.\rightarrow 1_{1}^{+}\right)=0.5 \mu_{N}^{2}$. The state vector contains proton 2QP spin-flip components connecting the spin-orbit partners of the $2 d$ shell $(99 \%)$ with a minor admixture of $1 h$-shell contributions $(0.2 \%)$. The most significant part of the $M 1$ strength below the particle threshold is given by the $1_{2}^{+}$state at $E^{*}=6.43 \mathrm{MeV}$ with $B\left(M 1\right.$; g.s. $\left.\rightarrow 1_{2}^{+}\right)=2.4 \mu_{N}^{2}$, where the most important proton 2QP components result from spin flip within the $1 \mathrm{~g}$ shell $(95.1 \%)$, and again with a small admixture from the neutron $1 h$ shell (4.8\%), respectively. A large $M 1$ strength associated with the maximum of the spin-flip M1 strength is found close to the neutron-separation energy at $E^{*}=8.7 \mathrm{MeV}$ (see as well in [30]) with $B\left(M 1 ;\right.$ g.s. $\left.\rightarrow 1_{3}^{+}=8 \mu_{N}^{2}\right)$. It essentially consists of the neutron 2QP spin-flip components in the $1 h$ shell (94\%) and a small amount of $1 g$-shell proton contributions (5\%).

In Fig. 3 multiphonon results for the electric and magnetic photoabsorption cross sections are presented in comparison to the present experimental data for the $1^{ \pm}$states in
${ }^{138} \mathrm{Ba}$ at $E^{*}<8.5 \mathrm{MeV}$. The measured elastic $E 1$ (open histogram) and $M 1$ (solid histogram) cross sections, integrated over $200 \mathrm{keV}$ bins, are shown in the upper part of Fig. 3. As can be seen at excitation energies below the particle emission threshold, the $E 1$ transitions in ${ }^{138} \mathrm{Ba}$ are distributed in a broad, resonancelike structure. The observed concentration of $1^{-}$states is characterized by the high level density which increases steeply towards the threshold. In this connection, the multiphonon QPM calculations which account for nonharmonic effects, are important for reproducing the fragmentation pattern. The calculations show that the $1^{-}$QRPA states are fragmented over 130 multiphonon states below $E^{*}=8.5 \mathrm{MeV}$. This agrees very well with the number of experimentally observed $1^{-}$states in this energy range. In contrast, the magnetic dipole transitions which result from the decay of single-particle states, are much more isolated and they are concentrated at well specified regions. For example, a group of $1^{+}$excited states, located at $E^{*}=6-7.5 \mathrm{MeV}$, is connected to the dissipation and fragmentation of the $1_{2}^{+}$ QRPA state. Above $8 \mathrm{MeV}$ the $1^{+}$states are characterized predominantly by multiphonon configurations, incorporating only a few percent of the strength of the $1_{3}^{+}$QRPA state (indicating the maximum of the QRPA $M 1$ strength). In the energy range $E^{*}=6-8.5 \mathrm{MeV} 191^{+}$states with transition strengths larger than $0.05 \mu_{N}^{2}$ have been observed. For comparison, theory predicts 15 such states.

In addition, the QPM calculations predict $M 1$ strengths smaller than $0.05 \mu_{N}^{2}$ and distributed in $581^{+}$states with excitation energies $E^{*}<8.5 \mathrm{MeV}$. In particular, an amount of this strength is concentrated around $4 \mathrm{MeV}$. These excitations are closely related to the fragmentation of the $1_{1}^{+}$QRPA state. The discrepancy between the pre-

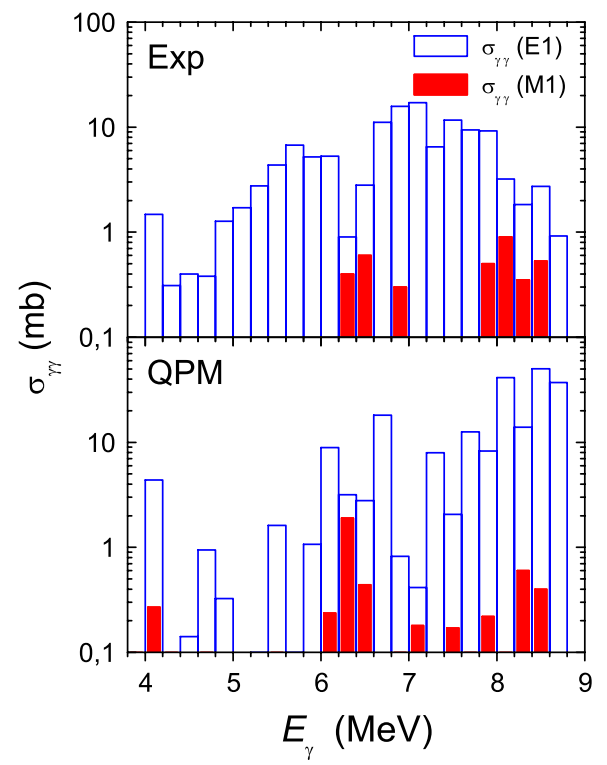

FIG. 3 (color online). Experimental (top panel) and QPM calculations (lower panel) for $E 1$ and $M 1$ elastic scattering cross sections in ${ }^{138} \mathrm{Ba}$ below $9.0 \mathrm{MeV}$. The cross sections are averaged over $0.2 \mathrm{MeV}$ energy bins. 
TABLE I. $\quad E 1$ and $M 1$ parameters deduced in ${ }^{138} \mathrm{Ba}$ below the neutron-separation energy in comparison with the QPM calculations.

\begin{tabular}{lccccc}
\hline \hline & $\left\langle E_{E 1}\right\rangle[\mathrm{MeV}]$ & $\Sigma B(E 1) \uparrow\left[e^{2} \mathrm{fm}^{2}\right]$ & $\left\langle E_{M 1}\right\rangle[\mathrm{MeV}]$ & $\Sigma B(M 1) \uparrow\left[\mu_{N}^{2}\right]$ & $\mathrm{EWSR}_{E 1}[\%]$ \\
Experimental & 6.7 & $0.96(18)$ & 6.9 & $2.5(6)$ & 1.3 \\
QPM & 7.3 & 1.22 & $6.9^{\mathrm{a}}$ & $2.9^{\mathrm{a}}$ & 1.8 \\
\hline \hline
\end{tabular}

a $4.1 \mathrm{MeV}<E^{*}<8.5 \mathrm{MeV}$.

dicted and observed $M 1$ strength shown in Fig. 3 below $6 \mathrm{MeV}$ can probably be attributed to local fragmentation of the strength into states that are too weak to be detected.

Overall, as summarized in Table I, the theoretical calculations are in good agreement with the present $E 1$ and $M 1$ data below the particle threshold, both with respect to the mean excitation energy, defined as $\langle E\rangle=\Sigma E_{i} B_{i} / \Sigma B_{i}$, total strength $\Sigma B_{i}$, and the isovector EWSR [17]. However, Fig. 3 and Table I also show that the theoretical strength distribution appears to be shifted upward by about $500 \mathrm{keV}$. This shift can be traced back to a slightly too large spacing of the theoretical 2QP spectrum, which in turn is probably caused by an underestimation of core polarization effects, although up to three phonon configurations are taken into account already. Polarization self-energies acting directly on the quasiparticle states may be required.

A common feature of $1^{-}$and $1^{+}$states is that both modes are excited by almost pure 2QP QRPA states. They serve as doorway states which decay into multiconfiguration states with complicated multiphonon wave functions, thus giving rise to fragmentation of the spectral distributions. Therefore, the fragmentation pattern, especially above $7 \mathrm{MeV}$, is not yet described in all details. However, the basic mechanism responsible for a more detailed fragmentation pattern is clear: it will eventually be accomplished by further increase of the multiphonon configuration space used in the QPM calculations.

The comparison of $E 1$ and $M 1$ strengths in the whole energy range between 4 and $8.6 \mathrm{MeV}$ shown in Fig. 3 leads to the conclusion that $E 1$ transitions clearly dominate $M 1$ transitions by intensity and amplitude. The ratio of elastic $M 1$ and $E 1$ cross sections is only $2.7(0.8) \%$. This ratio is in very good agreement $(2.0 \%)$ with the theoretical calculations based on the QPM model, and presented in the bottom panel of Fig. 3 .

In conclusion, the systematic spin and parity measurements on ${ }^{138} \mathrm{Ba}$ at the $\mathrm{HI} \vec{\gamma} \mathrm{S}$ facility have unambiguously verified that the observed dipole strength from $4 \mathrm{MeV}$ to the neutron-separation energy is predominantly of electric dipole nature. The fine structure of the $M 1$ "spin-flip" mode was detected as well, for the first time in $N=82$ nuclei. Enhanced dipole strength above the Lorentzian extrapolation of the GDR has been directly measured for elastic and inelastic transitions below the neutronseparation energy and found to be related to the dominance of electric transitions. This strength falls into two categories: elastic, originated by the skin oscillation of the excess neutrons against the proton-neutron saturated core, and inelastic, due to states coupled to more complicated con- figurations than $1 p-1 h$ states. Combining these two strengths, they define the gross structure of the PDR resonance which exhibits a resonancelike shape distribution with pronounced peak structure above the low-energy extrapolation of the GDR. According to the theoretical model, electric and magnetic transitions are found to be of single-particle character, but related to different physical mechanisms. In order to determine the pure dipole strength associated with the neutron skin phenomenon, the magnetic contribution must be identified and subtracted.

We are grateful to the $\mathrm{HI} \vec{\gamma} \mathrm{S}$ staff for their delivery of excellent photon beams. This research was supported by the DOE Grants No. DE-FG02-97ER41033, No. DEFG02-97ER41041, No. DE-FG02-97ER41042, No. DEFG52-06NA26155, and DFG Grant No. Le439/6.

[1] R.-D. Herzberg et al., Phys. Rev. C 60, 051307(R) (1999).

[2] N. Ryezayeva et al., Phys. Rev. Lett. 89, 272502 (2002).

[3] A. Zilges et al., Phys. Lett. B 542, 43 (2002).

[4] P. Adrich et al., Phys. Rev. Lett. 95, 132501 (2005).

[5] S. Volz et al., Nucl. Phys. A779, 1 (2006).

[6] D. Savran et al., Phys. Rev. Lett. 97, 172502 (2006).

[7] G. Rusev et al., Eur. Phys. J. A 27, 171 (2006).

[8] R. Schwengner et al., Phys. Rev. C 78, 064314 (2008).

[9] D. Savran et al., Phys. Rev. Lett. 100, 232501 (2008).

[10] O. Wieland et al., Phys. Rev. Lett. 102, 092502 (2009).

[11] Y. Suzuki et al., Prog. Theor. Phys. 83, 180 (1990).

[12] P. Van Isacker M. A. Nagarajan, and D. D. Warner, Phys. Rev. C 45, R13 (1992).

[13] N. Paar et al., Rep. Prog. Phys. 70, 691 (2007).

[14] N. Tsoneva, H. Lenske, and Ch. Stoyanov, Phys. Lett. B 586, 213 (2004).

[15] N. Tsoneva and H. Lenske, Phys. Rev. C 77, 024321 (2008).

[16] G. Tertychny et al., Phys. Lett. B 647, 104 (2007).

[17] M. N. Harakeh and A. van der Woude, Giant Resonances: Fundamental High-Frequency Modes of Nuclear Excitation (Oxford University Press, New York, 2001).

[18] M. Arnould and S. Goriely, Phys. Rep. 384, 1 (2003).

[19] T. Aumann, Eur. Phys. J. A 26, 441 (2005).

[20] U. Kneissl et al., Prog. Part. Nucl. Phys. 37, 349 (1996).

[21] N. Pietralla et al., Phys. Rev. Lett. 88, 012502 (2001).

[22] C. Fransen et al., Phys. Rev. C 70, 044317 (2004).

[23] T. C. Li et al., Phys. Rev. C 73, 054306 (2006).

[24] R. Longland et al., Phys. Rev. C 80, 055803 (2009).

[25] H. R. Weller et al., Prog. Part. Nucl. Phys. 62, 257 (2009).

[26] N. Pietralla et al., AIP Conf. Proc. 656, 365 (2003).

[27] N. Pietralla et al., Phys. Lett. B 681, 134 (2009).

[28] B. L. Berman et al., Phys. Rev. C 2, 2318 (1970).

[29] V.G. Soloviev, Theory of Complex Nuclei (Pergamon Press, Oxford, 1976).

[30] V. G. Soloviev et al., Phys. Lett. B 79, 187 (1978). 